\title{
Concentrating on intrinsic disorder
}

Many eukaryotic proteins contain intrinsically disordered regions (IDRs), which serve as flexible platforms for protein-protein interactions and can phase-separate into liquid-like condensates. Understanding the properties and functionality of IDR-based interactions is still in its infancy. Three independent studies published in Science indicate that dynamic IDR-IDR interactions can concentrate transcription regulators on DNA to establish a transcriptioncompetent compartment. Another study, reported in Cell, provides a comprehensive description of the molecular interactions that underlie the propensity of IDR-containing proteins to phase-separate.

Young and colleagues studied the role of IDRs in regulating superenhancers (SEs) - large clusters of enhancers that drive robust expression of cell identity genes and are characterized by high concentration of transcription regulators. They observed that two SE-associated transcription co-activators, bromodomaincontaining protein 4 (BRD4) and Mediator of RNA polymerase II transcription subunit 1 (MED1) formed distinct puncta in nuclei of murine embryonic stem cells (ESCs), which often colocalized at SEs. These puncta showed high protein turnover, akin to the dynamics of liquid-like

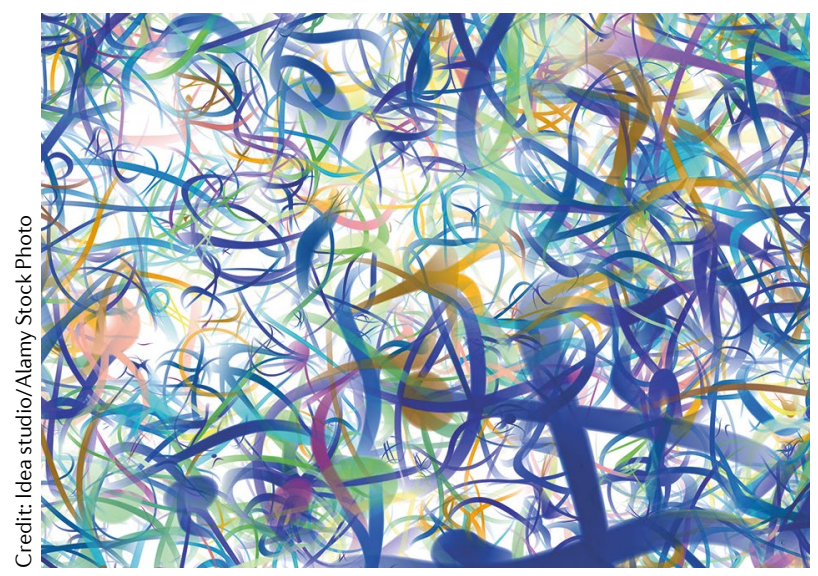

condensates. Furthermore, IDRs from BRD4 and MED1 formed

dynamic

IDR-IDR

interactions

can

concentrate

transcription

regulators

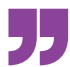

phase-separated condensates in vitro and in cells after optogenetically induced aggregation. Importantly, MED1-IDR condensates were capable of sequestering BRD4 and the largest subunit of RNA polymerase II (RNA Pol II), and inhibited transcription in nuclear extracts. Furthermore, formation of BRD4 and MED1-containing puncta was required for RNA Pol II enrichment at SEs in ESCs.

The formation of dynamic clusters of the Mediator complex on SEs that colocalized with RNA Pol II was visualized by Cisse and colleagues, using live cell super-resolution and lattice light sheet microscopy. Thus, IDRs can drive the condensation of transcription co-activators at SEs, which facilitates the recruitment and concentration of other components of the transcription machinery. Based on the in vitro properties of these IDRs and their dynamics in cells, it was proposed that the capacity of IDRs to undergo phase separation is the driving force for protein condensation at SEs.

Tjian and colleagues investigated the cellular dynamics of transcription factor-derived IDRs, which were targeted to endogenous or synthetic arrays of repetitive DNA. These IDRs formed nuclear puncta and recruited more IDRs through IDR-IDR interactions. These interactions were specific (not all IDRs could interact with each other) and dynamic. When highly expressed, IDRs formed puncta in the nucleoplasm independently of DNA, and in these nucleoplasmic puncta IDR-IDR interaction dynamics was even greater. Thus, repetitive DNA arrays promote the local concentration of DNA-targeted IDRs, such as those of transcription factors, thereby establishing IDR hubs that stabilize IDR-IDR interactions and facilitate further protein recruitment.
Importantly, these hubs could recruit RNA Pol II and were shown to be required for transcription factor function in gene expression. As IDR hubs consistently formed without noticeable phase separation, further studies are needed to clarify the function of phase separation in gene expression in vivo.

Inter-IDR interactions and condensate or hub formation were influenced by the amino acid composition of IDRs, raising the question of how the primary sequence of IDR-containing proteins controls their behaviour. This question was addressed by Hyman, Alberti and colleagues, who investigated the properties of 22 IDR-containing proteins of the FUS family. They found that only three of these proteins were capable of phase separation in vitro under physiologically relevant conditions. Electrostatic interactions between Tyr (in IDRs) and Arg (in RNA-binding domains) were the key drivers of phase separation. Inter-IDR Tyr-Tyr interactions could also promote phase separation, but only at higher protein concentrations. Other residues determined the properties of condensates, including fluidity (Gly) and hardening (Ser and Gln).

In the future it will be important to further dissect the role of discrete protein regions and amino acids in regulating IDR-containing protein interactions. It will also be interesting to establish how specificity is achieved in these very dynamic interactions, how they control protein compartmentalization in different cellular contexts and how phase separation contributes to these processes in vivo.

Paulina Strzyz

ORIGINAL ARTICLES Sabari, B. R. et al. Coactivator condensation at super-enhancers links phase separation and gene control. Science https://doi.org/10.1126/science.aar3958 (2018) | Cho, W.-K. et al. Mediator and RNA polymerase Il clusters associate in transcriptiondependent condensates. Science https://doi.org/ 10.1126/science.aar4199 (2018) |Chong, S. et al. Imaging dynamic and selective low-complexity domain interactions that control gene transcription. Science https://doi.org/10.1126/ science.aar2555 (2018) | Wang, J. et al. A molecular grammar governing the driving forces for phase separation of prion-like RNA binding proteins. Cell https://doi.org/10.1016/j.cell.2018.06.006 (2018) FURTHER READING Banani, S. F.et al. Biomolecular condensates: organizers of cellular biochemistry. Nat. Rev. Mol. Cell Biol. 18, 285-298 (2017) 